\title{
ESTABLECIMIENTO DE UN VALOR ADICIONAL DE PUNTO DE ROCIO DE HIDROCARBURO PARA LA ZONA CÁLIDA DE COLOMBIA
}

\author{
Nicolás Santos Santos*; Julio Cesar Pérez Angulo; Manuel Carbarcas Simancas. \\ Grupo de Modelamiento de Procesos de Hidrocarburos. Universidad Industrial de Santander (UIS). Carrera 27 Calle 9. \\ Bucaramanga. Colombia. \\ *E-mail: nicolas@uis.edu.co
}

\section{RESUMEN}

El contenido de pequeñas cantidades de agua y condensados en el gas natural generan problemas en el transporte por líneas, causan corrosión y otros daños en equipos de compresión o turbinas. Es por esto que el gas natural se debe someter a diversos tratamientos para cumplir con las especificaciones de calidad establecidas, entre los parámetros de calidad está el Punto de Rocío de Hidrocarburo (HCDP), el cual indica la temperatura mínima a la cual puede estar el gas sin que se presenten condensados. Para establecer este parámetro, es necesario tener en cuenta criterios termodinámicos, climáticos y regulatorios. Pero no todas las regulaciones de HCDP son iguales, por consiguiente, un gas que es transportado en la red de gasoductos de un país puede no ser apto para la red de otro país. Dado lo anterior, en el presente artículo se presenta el establecimiento de un posible valor adicional de punto de rocío de hidrocarburo para la zona cálida de Colombia, realizando una simulación hidráulica en estado dinámico que contempla la inclusión del perfil de temperatura y el relieve que atraviesa el gasoducto.

Palabras clave: HCDP, condensados, punto de rocío por hidrocarburo, gas natural.

\section{ESTABLISHMENT OF AN ADDITIONAL VALUE OF HCDP IN THE WARM COLOMBIAN ZONE}

\begin{abstract}
The content of small amounts of water and condensate in the natural gas generate problems in transport lines, cause corrosion and other damage in compression equipment or turbines. That is why natural gas must be submitted to various treatments to meet established quality specifications; between the quality parameters is the Hydrocarbon Dew Point (HCDP), which indicates the minimum temperature at which gas can be submitted without condensed, to set this parameter, it is necessary to consider thermodynamic, climate and regulatory criteria. However, not all HCDP regulations are equal; therefore, a gas that is transported in the pipeline system of a country may not be suitable for the network in another country. Given the above, this article presents the establishment of a possible additional value hydrocarbon dew point for the warm area of Colombia, performing a hydraulic simulation in dynamic state, which provides for inclusion of the temperature profile and the relief flowing through the pipeline.
\end{abstract}

Keywords: HCDP, Condensate, Hydrocarbon dew Point, natural gas.

Cita: Santos, N., Pérez, J. y Carbarcas, M. (2017). Establecimiento de un valor adicional de punto de rocio de hidrocarburo para la zona cálida de Colombia. Revista Fuentes: El reventón energético, 15 (1), 59-73. 


\section{INTRODUCCIÓN}

El gas natural se debe someter a diversos tratamientos con el fin de cumplir las especificaciones de calidad establecidas por los diferentes organismos de control y regulación, ya que las líneas de transporte de gas natural y ciertos equipos están diseñados para trabajar con fluidos en una sola fase, es por eso que procesos como la deshidratación y control del Punto de Rocío Hidrocarburo (HCDP), resultan indispensables para prevenir futuros problemas operacionales. La eliminación del contenido de agua en un gas evita la reducción de la capacidad de flujo del gas, corrosión, y en caso de que la temperatura sea demasiado baja que se presente interrupción de flujo o taponamiento, además de otros problemas relacionados con la formación de hidratos. Cuando no existe un adecuado control del punto de rocío de hidrocarburo, los condensados se acumulan en la sección inferior de la tubería, además ocasionan inconvenientes en las instalaciones del sistema, como en los compresores y finalmente pueden ocasionar incendios y explosiones.

Por esto es necesario tener un control especial con el punto de rocío de Hidrocarburo, considerando los diferentes factores usados para establecerlo, como: composición del gas, condiciones de operación del gasoducto, condiciones climáticas y ambientales de la zona de operación y las principales normas de calidad establecidas por los diferentes organismos.

En el presente artículo se busca revisar y analizar la información correspondiente a la determinación del punto de rocío de hidrocarburo a nivel internacional, señalando los factores necesarios para dicha determinación, de tal manera que permita crear un marco de referencia para evaluar la factibilidad de implementar un valor adicional de punto de rocío de Hidrocarburo para la zona cálida de Colombia, con base en los criterios empleados en países con climas similares, realizando una simulación hidráulica en estado dinámico que contempla la inclusión del perfil de temperatura y el relieve que atraviesa el gasoducto.

\section{CRITERIOS USADOS A NIVEL INTERNACIONAL PARA ESTABLECER EL HCDP}

El transporte de gas por gasoductos necesita condiciones de operación que garanticen seguridad, brinden confiabilidad a los integrantes de la cadena del gas natural y aseguren la integridad de los equipos de las compañías transportadoras. Razón por la cual existen regulaciones o normatividades que establecen especificaciones de calidad que debe cumplir el gas durante todo su recorrido en el sistema de transporte. Uno de los parámetros más importantes de estas regulaciones es el punto de rocío de hidrocarburos (Hydrocarbon Dew Point - HCDP), el cual indica la temperatura mínima a la cual puede estar el gas sin que se presente condensación de líquidos hidrocarburos en el sistema de gasoductos.

Para establecer este parámetro es necesario tener en cuenta una serie de criterios termodinámicos, climáticos $\mathrm{y}$ regulatorios que en conjunto permiten realizar una determinación de estándares de calidad que contribuyan a un adecuado desarrollo del mercado del gas natural.

Así mismo, es importante resaltar que no todas las regulaciones de HCDP son iguales y en consecuencia, un gas que es transportado en la red de gasoductos de un país puede que no sea apto para ser transportado en otro. A continuación, se presentan la formas en la cuales se puede encontrar especificado el HCDP.

\subsection{FORMAS DE ESPECIFICAR EL PUNTO DE ROCIO DE HIDROCARBUROS}

La especificación del punto de rocío de hidrocarburos (HCDP), puede tomar una de las siguientes tres formas, una es cuantitativa y las otras dos son cualitativas (Agency for the Cooperation of Energy Regulators [ACER], 2011).

\subsubsection{FORMA CUANTITATIVA}

Se especifica un valor de temperatura máximo para un rango de presión. Por ejemplo, la norma de calidad del gas en Francia establece un HCDP de $-2^{\circ} \mathrm{C}$ entre 1-70 bar de presión $\left(28.4^{\circ} \mathrm{F} @ 14.2\right.$ - 994.5 psi) (ACER, 2011).

\subsubsection{Formas CuAlitativas}

El HCDP establecido de esta forma se presenta de las siguientes maneras:

- "Temperatura Ambiente para todas las presiones" (Ground Temperature for all Pressures). Esta declaración generalmente comprende un valor de - $4^{\circ} \mathrm{C}\left(24.8^{\circ} \mathrm{F}\right)$. Alemania es un ejemplo de esta forma de especificar el HCDP (Alemania-GRTgaz, 2013).

- El gas se encuentra "Técnicamente libre de Condensados" (...Technically free of...) tal como se puede ver en la norma de calidad del gas de Reino Unido (POYRY, 2011). 


\subsection{CRITERIOS TERMODINÁMICOS}

El comportamiento termodinámico del gas natural está directamente relacionado con su composición, lo cual convierte los criterios termodinámicos en parte fundamental para la especificación de calidad del gas natural (por ejemplo, el poder calorífico, comportamiento de fases, punto de rocío de hidrocarburos, número Wobby, etc) (MARCOGAZ, 2003-2002).

Un ejemplo de la importancia del comportamiento termodinámico del gas para establecer la especificación de calidad del gas natural tiene lugar en Europa, donde existen dos categorías de suministro de gas: el gas de bajo poder calorífico (Gas L) y de alto poder calorífico (Gas H). Estos gases presentan diferencias en sus especificaciones de calidad lo que produce restricciones de interoperabilidad, lo cual se puede definir como, la capacidad de un producto a interactuar con otros de sus mismas características, es decir, los gases L y $\mathrm{H}$ no se pueden mezclar y por lo tanto son suministrados a través de redes de transporte independientes (MARCOGAZ, 2003).

Los Países Bajos son la única fuente de Gas-L, mientras que el Gas-H tiene numerosos orígenes, entre ellos: Mar del Norte, Rusia, Turkmenistán, entre otros. En cuanto al sistema de transporte, Gas-H está presente en toda Europa, mientras que el Gas-L es distribuido únicamente en cuatro países: Francia, Bélgica, Países Bajos y una pequeña parte de Alemania (MARCOGAZ, 2002).

\subsection{CRITERIOS CLIMÁTICOS}

El comportamiento climático y el relieve de la zona o región que atraviesa el gasoducto es determinante para establecer el HCDP, ya que, pueden existir puntos con temperaturas muy bajas que podrían generar condensación. Las bajas temperaturas pueden estar asociadas a la existencia de pisos térmicos y/o estaciones a lo largo del trazado del gasoducto (ACER, 2011).

Ya que la determinación del punto de rocío de hidrocarburos (HCDP) está basada en la interacción del gas natural que fluye dentro del gasoducto y su medio ambiente, es necesario realizar una simulación hidráulica en estado dinámico que contemple la inclusión del perfil de temperatura y el relieve que atraviesa el gasoducto.

\subsection{CRITERIOS REGULATORIOS}

Las normas de calidad del gas no cubren los mismos parámetros en todos los países donde existe infraestructura de transporte de gas natural. Las especificaciones de calidad del gas pueden ser decretadas por el gobierno de cada país, en cabeza del organismo de control de transporte de gas, o pueden ser regulaciones establecidas de manera privada e independiente por las empresas productoras y transportadoras vinculadas a un contrato de gas en particular.

De acuerdo a la reglamentación de cada país, sea regulación nacional (legislativa) o especificación del sistema de transporte (contractual), se puede presentar alguna de las siguientes modalidades de HCDP: (1) Modalidad de Valor Único de HCDP, (2) Modalidad Contractual, (3) Modalidad Eurozona y (4) Modalidad de Múltiple Punto de Rocío (POYRY, 2011).

\subsubsection{MODALIDAD UNICO VALOR DE HCDP}

En los países con un valor único de HCDP, la especificación del punto de rocío es de carácter legislativo y se encuentra regulada por organismos del gobierno como Ministerios o Agencias de Energía, las cuales buscan promover la uniformidad de la toda la cadena productiva del gas natural.

En la Tabla 1 se presenta un resumen de los aspectos más importantes de las regulaciones de los países con un único valor de HCDP. De igual forma, aunque la mayoría de países europeos tienen un único valor de HCDP, estos serán discutidos bajo la modalidad de Eurozona (BP. International Gas Union, 2011).

Tabla 1. Resumen Países con Valor Único de HCDP (Argentina-ENARGAS, 2005), (Bolivia-Superintendencia de Hidrocarburos 2007), (Ecuador-Instituto Ecuatoriano de Normalización, 2009), (México. Comisión Reguladora de Energía.), (Nueva Zelanda. Gas Industry Company Limited)

\begin{tabular}{|c|c|c|c|c|}
\hline País & Norma & Ente de Control & $\begin{array}{l}\text { HCDP } \\
\text { (S.I) }\end{array}$ & $\begin{array}{l}\text { Método de } \\
\text { Medición }\end{array}$ \\
\hline Colombia & RUT & CREG & $45^{\circ} \mathrm{F}$ & \\
\hline Argentina & Res 622/98 & ENARGAS & $\begin{array}{c}24.8^{\circ} \mathrm{F} \\
@ 798 \mathrm{psi}\end{array}$ & $\begin{array}{l}\text { GPA } 2286 \\
\text { EOS PR }\end{array}$ \\
\hline Bolivia & $\begin{array}{c}\text { Res } \\
0433 / 2007\end{array}$ & $\begin{array}{l}\text { Superintendencia } \\
\text { de Hidrocarburos }\end{array}$ & $\begin{array}{l}32^{\circ} \mathrm{F} @ \\
640 \mathrm{psi}\end{array}$ & $\begin{array}{l}\text { Cromatografía } \\
\text { en Línea }\end{array}$ \\
\hline Ecuador & $\begin{array}{c}\text { NTE INEN } \\
2\end{array}$ & INEN & $\begin{array}{c}28.4^{\circ} \mathrm{F} @ \\
0-1160 \\
\text { psi }\end{array}$ & $\begin{array}{c}\text { ASTM D1142 } \\
\text { ASTM D1945 } \\
\text { ISO } 6975\end{array}$ \\
\hline Venezuela & $\begin{array}{c}\text { Norma } \\
\text { Venezolana } \\
\text { COVENIN }\end{array}$ & $\begin{array}{l}\text { Ministerio de } \\
\text { Minas y Energía }\end{array}$ & $35.6^{\circ} \mathrm{F}$ & $\begin{array}{l}\text { GPA } 2286 \\
\text { ISO } 6975\end{array}$ \\
\hline México & $\begin{array}{l}\text { NOM-001- } \\
\text { SECRE- } \\
2010\end{array}$ & $\begin{array}{c}\text { Comisión } \\
\text { Reguladora de } \\
\text { Energía }\end{array}$ & $28.4^{\circ} \mathrm{F}$ & \\
\hline $\begin{array}{l}\text { Nueva } \\
\text { Zelanda }\end{array}$ & $\begin{array}{c}\text { NZS } \\
5442: 1999\end{array}$ & & $\begin{array}{c}28.4^{\circ} \mathrm{F} @ \\
725 \mathrm{psi}\end{array}$ & \\
\hline $\begin{array}{l}\text { Emiratos } \\
\text { Árabes }\end{array}$ & & $\begin{array}{l}\text { Abu Dhabi } \\
\text { National Oil } \\
\text { Company - }\end{array}$ & $\begin{array}{c}28.4^{\circ} \mathrm{F} @ \\
14.5-1000 \\
\text { psi }\end{array}$ & \\
\hline
\end{tabular}




\subsubsection{MODALIDAD CONTRACTUAL}

Esta modalidad agrupa los países en los cuales las compañías transportadoras tienen la libertad de establecer la norma de calidad del gas que reciben y transportan por sus gasoductos; sin embargo, estas regulaciones deben ajustarse a estatutos de seguridad de cada país. En esta modalidad de HCDP, la compañía transportadora tiene la autoridad para no recibir el gas que no cumpla con las especificaciones, asegurando de esta manera que no haya presencia de líquidos en el gasoducto y garantizando la integridad de sus equipos. Algunos países con esta modalidad son Canadá, Estados Unidos, y Australia (Canadá, TransCanada 2006; 2013), (USA, The University of Center For Energy Economics, 2004).

En la Tabla 2, Se presentan los valores de HCDP establecidos en Canadá.

En la Tabla 3, se presenta una recopilación del HCDP dispuesto en las especificaciones de calidad del gas de las principales compañías transportadoras de gas en los Estados Unidos (USA, The University of Center For Energy Economics, 2004).

Tabla 2. Resumen especificación de HCDP en Canadá. (Canadá, TransCanada 2006; 2013).

\begin{tabular}{cc}
$\begin{array}{c}\text { SISTEMA DE } \\
\text { TRANSPORTE } \\
\text { (GASODUCTO) }\end{array}$ & HCDP \\
\hline $\begin{array}{c}\text { Canadian Mainline } \\
\text { System }\end{array}$ & $-10^{\circ} \mathrm{C}\left(14^{\circ} \mathrm{F}\right) @ 5500 \mathrm{kPa}$ Abs \\
NTGL System & $-10^{\circ} \mathrm{C}\left(14^{\circ} \mathrm{F}\right) @$ Presión de \\
Operación
\end{tabular}

Tabla 3. Resumen especificación de HCDP en Estados Unidos (USA, The University of Center For Energy Economics, 2004).

\begin{tabular}{|c|c|}
\hline $\begin{array}{l}\text { SISTEMA DE TRANSPORTE } \\
\text { (GASODUCTO) }\end{array}$ & HCDP \\
\hline Alliance USA & 14F @ Presión de Operación \\
\hline Empire State Pipeline & $\mathrm{N} / \mathrm{E}$ \\
\hline $\begin{array}{c}\text { Great Lakes Gas Transmission } \\
\text { GLGT }\end{array}$ & $\mathrm{N} / \mathrm{E}$ \\
\hline $\begin{array}{c}\text { Iroquois Gas Transmission } \\
\text { System }\end{array}$ & $15^{\circ} \mathrm{F}$ ó menos \\
\hline Northern Border & $\begin{array}{l}\mathrm{HCDP}_{1}=-5^{\circ} \mathrm{F} @ 800 \mathrm{psia} \\
\mathrm{HCDP}_{2}=-10^{\circ} \mathrm{F} @ 800 \mathrm{psia} \\
\mathrm{HCDP}_{3}=-18^{\circ} \mathrm{F} @ 800 \mathrm{psia}\end{array}$ \\
\hline North West Pipeline NWP & $15^{\circ} \mathrm{F} @ 100-1000$ psia \\
\hline PNGTS & $\mathrm{N} / \mathrm{E}$ \\
\hline SOCAL & $\mathrm{N} / \mathrm{E}$ \\
\hline Tennessee GP & $15^{\circ} \mathrm{F}$ \\
\hline Viking Gas Transmission & $\mathrm{N} / \mathrm{E}$ \\
\hline Northern Natural Gas Co. & $\mathrm{N} / \mathrm{E}$ \\
\hline Pacific Gas Transmission Co. & $-9.5^{\circ} \mathrm{C} @ \mathrm{P}>55$ bar \\
\hline Transwestern Pipeline Gas Co. & Libre de Líquidos \\
\hline Florida Gas Transmission Co. & $\mathrm{N} / \mathrm{E}$ \\
\hline Southwest U. S. Company & $\mathrm{N} / \mathrm{E}$ \\
\hline Midwest U. S. Company & $\mathrm{N} / \mathrm{E}$ \\
\hline Southeast U. S. Company & $\mathrm{N} / \mathrm{E}$ \\
\hline
\end{tabular}

De acuerdo a la evidencia regulatoria mostrada anteriormente y al gran mercado binacional de gas existente entre Canadá y Estados Unidos, es viable considerar estos dos países como una sola región con la presencia de múltiples puntos de rocío de hidrocarburos.

\subsubsection{MODALIDAD \\ ESPECIAL: EUROZONA}

Europa es el más claro ejemplo de la globalización del mercado del gas natural debido al continuo crecimiento de la demanda de combustible. En Europa existen gasoductos que atraviesan prácticamente todo el continente suministrando gas natural que es producido en cierta región de un país a otros países. Debido a esto es posible analizar todo el continente europeo como si fuera una sola región con una amplia red de gasoductos para el transporte de gas natural con múltiples valores de punto de roció de hidrocarburos (HCDP) (MARCOGAZ, 2003-2002).

Especificaciones de Calidad del Gas natural de Europa. El mercado de gas natural europeo contempla la existencia de regulaciones nacionales en países con valor único de HCDP, al mismo tiempo que existen países con modalidad contractual ejercida por cada empresa 
transportadora. Sin embargo, la presencia de gasoductos internacionales que atraviesan más de un país hace necesaria la existencia de una regulación que permita la interoperabilidad, es decir, el gas que es transportado por un gasoducto en un país cumpliendo la respectiva regulación puede que no sea apto para transporte según la especificación de otro país, razón por la cual es necesario establecer un valor HCDP que satisfaga la condición de interoperabilidad (MARCOGAZ, 2003).

De acuerdo a lo anterior, es posible encontrar países con redes de transportes locales con valores de HCDP diferentes a los valores de los gasoductos internacionales, lo cual valida la premisa presentada anteriormente de analizar todo el continente europeo como una sola región (eurozona) con múltiples puntos de rocío dado también la existencia de estaciones (MARCOGAZ, 2003), (POYRY, 2011).

En la Tabla 4, se presenta la recopilación de la información regulatoria de HCDP de Europa.

\subsubsection{MODALIDAD MULTIPLE PUNTO DE ROCÍO}

Brasil, La Agencia Nacional de Petróleo ANP establece por medio de la Resolución ANP No. 16 de 2008, el reglamento técnico para la especificación del gas natural, nacional o importado, que se venden en todo Brasil. Esta regulación establece especificaciones para tres zonas en las que se agrupan las cinco regiones geopolíticas de Brasil, donde cada zona tiene un valor de HCDP y son presentados en la Tabla 5 (Brasil-ANP 2002-2008).

Como se puede apreciar, existen dos valores diferentes de puntos de roció de hidrocarburos (HCDP), los cuales fueron establecidos de acuerdo a las zonas climáticas de Brasil.

Australia, Debido a la gran diversidad climática y el sobresaliente desarrollo económico e industrial, Australia, cuenta con un sistema de transporte de gas natural compuesto por aproximadamente 64 gasoductos. En cuanto a la regulación de especificación de calidad del gas natural, Australia presenta múltiples valores de HCDP (GREAT SOUTHERN PRESS. Australia). y tiene un control polivalente ya que una parte del sistema de transporte es regido por normas establecidas por el gobierno australiano en cabeza de los diferentes organismos de control del mercado del gas natural, mientras que la otra parte del sistema es controlada de manera contractual y las especificaciones son establecidas por cada compañía transportadora.
Tabla 4. Resumen Especificación de Calidad del Gas en Europa (Alemania-GRTgaz, 2013), (Belgica, 2004), (España-Enagas, 2004), (Irlanda, 2008), (Lituania, 2012), (Nueva Zelanda, 1999), (Poyry, 2011), (Rusia, 2008), (United Kingdom Interconnector 2013), (United Kingdom. Premier Transmission Limited.)

\begin{tabular}{|c|c|c|c|}
\hline País & $\begin{array}{c}\text { Norma o } \\
\text { Código }\end{array}$ & $\begin{array}{l}\text { Ente de } \\
\text { Control }\end{array}$ & $\begin{array}{l}\text { HCDP } \\
\text { (SI) }\end{array}$ \\
\hline \multirow[t]{2}{*}{ UK } & $\begin{array}{c}\text { Gas Safety } \\
\text { (Management) } \\
\text { Regualtions } \\
1996\end{array}$ & $\begin{array}{l}\text { Comité de } \\
\text { Salud y } \\
\text { Seguridad }\end{array}$ & $\begin{array}{c}\text { Libre de } \\
\text { Hidrocarburos } \\
\text { condensables }\end{array}$ \\
\hline & Valores Típicos & Ley Local & $\begin{array}{c}28.4^{\circ} \mathrm{F} @ \mathrm{P}>=1232 \\
\mathrm{psi}\end{array}$ \\
\hline Alemania & $\begin{array}{l}\text { DVGW Coe of } \\
\text { Practice G } 260\end{array}$ & DVGW & $\begin{array}{c}\text { Temperatura } \\
\text { ambiente a presión } \\
\text { de operación }\end{array}$ \\
\hline Austria & & & 32 F@ O.P. \\
\hline Bélgica & & & $32^{\circ} \mathrm{F} @$ O.P. \\
\hline Dinamarca & $\begin{array}{l}\text { Reglas para el } \\
\text { Transporte de } \\
\text { gas }\end{array}$ & Energinet.dk & $\begin{array}{c}24.8^{\circ} \mathrm{F} @ \mathrm{P}<=1015 \\
\text { psi }\end{array}$ \\
\hline Eslovaquia & & & 32 F @ O.P. \\
\hline Eslovenia & & & $23 @ 566$ psi \\
\hline España & $\begin{array}{c}\text { BOE, Abril } 4 \text { - } \\
2006\end{array}$ & STM & $\begin{array}{c}41^{\circ} \mathrm{F} @ \mathrm{P}<=1015 \\
\mathrm{psi}\end{array}$ \\
\hline Francia & & $\begin{array}{l}\text { Compañía de } \\
\text { Transporte }\end{array}$ & $\begin{array}{c}28.4^{\circ} \mathrm{F} @ \mathrm{P}<=1015 \\
\mathrm{psi}\end{array}$ \\
\hline Grecia & & & $37.4^{\circ} \mathrm{F} @ 1160$ psi \\
\hline Irlanda & & & $\begin{array}{c}28.4^{\circ} \mathrm{F} @ \mathrm{P}<=1232 \\
\mathrm{psi}\end{array}$ \\
\hline Italia & $\begin{array}{l}\text { Legislación } \\
\text { Nacional }\end{array}$ & $\begin{array}{l}\text { SNAM Rete } \\
\text { Gas }\end{array}$ & $32^{\circ} \mathrm{F}$ \\
\hline Luxemburgo & Easee-gas & & $28.4^{\circ} \mathrm{F}$ \\
\hline Noruega & $\begin{array}{c}\text { Términos y } \\
\text { condiciones para } \\
\text { el transporte } \\
\text { de gas }\end{array}$ & $\begin{array}{l}\text { Ministerio } \\
\text { del petróleo y } \\
\text { energía }\end{array}$ & $14^{\circ} \mathrm{F} @ 740 \mathrm{psi}$ \\
\hline Países Bajos & $\begin{array}{c}\text { Especificación } \\
\text { de Gas de } \\
\text { Entrada }\end{array}$ & $\begin{array}{l}\text { Gas Transport } \\
\text { Services }\end{array}$ & $26.6 @$ O.P. \\
\hline $\begin{array}{l}\text { Republica } \\
\text { Checa }\end{array}$ & & & $32^{\circ} \mathrm{F}$ \\
\hline Rumania & & & 32F@ O.P. \\
\hline Rusia & & & $28.4^{\circ} \mathrm{F} @ \mathrm{P}<=$ \\
\hline Suecia & & & $26.6^{\circ} \mathrm{F} @ 1160$ psi \\
\hline Suiza & G 2007/1 & SVGW & \\
\hline EASEE-gas & $\begin{array}{l}\text { Harmonisation } \\
\text { of Gas Qualities }\end{array}$ & & $\begin{array}{c}28.4^{\circ} \mathrm{F} @ \mathrm{P}<=1015 \\
\mathrm{psi}\end{array}$ \\
\hline
\end{tabular}

Tabla 5. Especificación de HCDP en Brasil (Brasil-ANP 2002-2008).

\begin{tabular}{ccccc} 
& \multicolumn{3}{c}{ Región } & Método de \\
\cline { 2 - 4 } Especificación & Norte & Noreste & $\begin{array}{c}\text { Medio oeste, } \\
\text { Sureste, Sur }\end{array}$ & Medición \\
\cline { 2 - 4 } HCDP & $15^{\circ} \mathrm{C}$ & $15^{\circ} \mathrm{C}$ & $0^{\circ} \mathrm{C}$ & ISO 6570 \\
\hline & $59^{\circ} \mathrm{F}$ & $59^{\circ} \mathrm{F}$ & $32^{\circ} \mathrm{F}$ & \\
\hline
\end{tabular}


Australia contempla tres valores diferentes de HCDP para su territorio. En la parte Oeste (Western Australia) que se caracteriza por ser principalmente de carácter desértico, se tiene un $\mathrm{HCDP}$ de $0^{\circ} \mathrm{C}\left(32^{\circ} \mathrm{F}\right)$. La zona norte (Northern Territory, Queensland) presenta gran variedad climática que van desde zonas desérticas, praderas, clima tropical e incluso una pequeña parte sujeta a clima ecuatorial por lo cual se establece un $\mathrm{HCDP}$ de $10^{\circ} \mathrm{C}\left(50^{\circ} \mathrm{F}\right)$ para esta zona. Por último la zona sur (South Australia, New South Wales, Victoria) presenta un comportamiento climático templado y por lo tanto tiene un HCDP de $2^{\circ} \mathrm{C}\left(35.6^{\circ} \mathrm{F}\right)$. (Great Australian Pipeline Industry Association).

\section{CRITERIOS DE REFERENCIA PARA ESTABLECER UN HCDP ADICIONAL EN COLOMBIA}

Una vez recopilada la información que comprende este marco de referencia, se procedió a establecer cuál de las modalidades anteriores es más acorde a la iniciativa para establecer un valor adicional de HCDP para la zona cálida colombiana. Para establecer esta referencia es necesario relacionar las posibles ventajas y desventajas de cada modalidad de HCDP presentadas y seleccionar aquella modalidad que se ajuste a los criterios climáticos y regulatorios existentes en Colombia (tabla 6).

Como se puede ver en la tabla anterior, el modelo de múltiple puntos de rocío es viable para Colombia, además es importante resaltar, que en el caso de Brasil, el valor de HCDP definido para la zona cálida es superior al valor establecido en la regulación actual colombiana, lo cual permite establecer la hipótesis de la factibilidad de definir un valor adicional para la zona cálida colombiana superior al valor actual. Sin embargo, es necesario desarrollar el debido proceso de investigación que permita validar esta hipótesis.

Tabla 6. Criterios de referencia para Colombia

\begin{tabular}{|c|c|c|c|c|}
\hline Modalidad & Zona Climática & Ventaja & Desventaja & Referencia para Colombia \\
\hline $\begin{array}{l}\text { Valor Único de } \\
\text { HCDP }\end{array}$ & $\begin{array}{c}\text { No hace diferencia entre } \\
\text { pisos térmicos }\end{array}$ & Uniformidad de mercado & $\begin{array}{l}\text { Diferencias entre compañías } \\
\text { productoras y transportadoras }\end{array}$ & Regulación Actual \\
\hline $\begin{array}{l}\text { Modalidad } \\
\text { Contractual }\end{array}$ & Zonas templadas y frías & $\begin{array}{l}\text { Compañía trasportadora con } \\
\text { libertad de establecer HCDP }\end{array}$ & $\begin{array}{l}\text { Productores que suministran gas } \\
\text { a gasoductos independientes }\end{array}$ & $\begin{array}{c}\text { Inviable ya que no existe } \\
\text { uniformidad de criterios entre } \\
\text { productores, transportadores y } \\
\text { entes reguladores }\end{array}$ \\
\hline $\begin{array}{l}\text { Modalidad } \\
\text { Eurozona }\end{array}$ & Zonas templadas y frías & Interoperabilidad & $\begin{array}{l}\text { Aplica únicamente para sistemas } \\
\text { de transmisión internacionales }\end{array}$ & $\begin{array}{c}\text { Inviable por falta de } \\
\text { infraestructura ya que el } \\
\text { mercado de gas colombiano, es } \\
\text { en su mayoría de carácter local }\end{array}$ \\
\hline $\begin{array}{l}\text { Múltiple Punto de } \\
\text { Rocío }\end{array}$ & $\begin{array}{l}\text { Tiene en cuenta los } \\
\text { diferentes pisos térmicos }\end{array}$ & $\begin{array}{l}\text { Puntos de rocío en función de } \\
\text { cada piso térmico }\end{array}$ & $\begin{array}{l}\text { Aplica para zonas con gran } \\
\text { diversidad climática }\end{array}$ & $\begin{array}{l}\text { Viable ya que proporciona } \\
\text { valores de HCDP para cada } \\
\text { zona de interés. }\end{array}$ \\
\hline
\end{tabular}

Así mismo, una vez revisada la información regulatoria de los países seleccionados, se encontró que la mayoría de regulaciones internacionales tienen especificado el parámetro del HCDP en función del punto de rocío, que al mismo tiempo está en función del piso térmico y no en función del Cricondentérmico (Cricondentherm), como está establecido en el Reglamento Único de Transporte de Colombia.

\subsection{MODELAMIENTO DE LA RED DE GASODUCTOS DE LA ZONA CÁLIDA COLOMBIANA}

Para el estudio de viabilidad hidráulica y termodinámica del valor de HCDP, es necesario definir los modelos de los gasoductos de PROMIGAS y TGI (sección
Ballena-Barrancabermeja). Para ello se debe disponer de información confiable de estaciones de compresión, topografía del recorrido del gasoducto, temperaturas ambientes mínimas, tipo de terreno, puntos de conexión a líneas de distribución urbana y rural (troncales), entre otros aspectos.

\subsubsection{MODELO DE SIMULACIÓN DE TGI (TRAMO BALLENA-BARRANCABERMEJA)}

El gasoducto Ballena-Barrancabermeja es un sistema de propiedad y operado por la Transportadora de Gas Internacional - TGI S.A. E.S.P., que atraviesa los departamentos de La Guajira, Magdalena, Cesar y Santander. Tiene una extensión de $578 \mathrm{Km}$ con tubería de 18 pulgadas con espesor de 0,344 pulgadas y calidad 
API 5LX-60, diseñado para una capacidad total de transporte de 260 MMSCFD. (Mapa red nacional de gasoductos: Gasoducto Ballena - Barrancabermeja). Actualmente cuenta con 6 estaciones de compresión (Hato Nuevo, La Jagua del Pilar, Casacará, Curumaní, Norean y San Alberto) operando a 1200 psig de presión de descarga y $120{ }^{\circ} \mathrm{F}$ de temperatura de descarga después de atravesar los aero-enfriadores respectivos (Mapa red nacional de gasoductos: Gasoducto Ballena - Barrancabermeja).

Para el diseño del modelo de simulación se sectorizó por tramos comprendidos entre cada estación de compresión. Éstos a su vez se dividieron en trayectos de distancias más cortas y variaciones de elevación más pequeñas que permitieran obtener resultados más detallados.

Los resultados fueron corroborados con el modelo de simulación de Peña (Peña, Santos y Pérez, 2001) sobre el mismo gasoducto, en las Tablas 7, 8, 9, 10, 11, 12 y 13 se tienen los datos de distancia y elevación por cada tramo. Para el modelo de simulación en específico, entre tramo y tramo, se ubicaron los depuradores de entrada a los respectivos compresores.

Tabla 7. Entrada de secciones, accesorios, elevación y longitud de tubería del tramo Ballena - Hato Nuevo de TGI (Ballena-Barrancabermeja).

\begin{tabular}{|ccccccc}
\hline & & & & & \\
\hline Pipe & 17,8 & 24,9 & 7,61 & 82 & 58399 & 0,08 \\
\hline Pipe & 26,3 & 49,9 & 15,23 & 82 & 27887 & 0,17 \\
\hline Fitting & 26,3 & 49,9 & 15,23 & 0 & 0 & 0 \\
\hline Pipe & 53,4 & 84,9 & 25,9 & 114 & 88976 & 0,07 \\
\hline Pipe & 53,9 & 99,9 & 30,47 & 49 & 1575 & 1,8 \\
\hline Pipe & 58,5 & 99,9 & 30,47 & 0 & 15091 & 0 \\
\hline Fitting & 58,5 & 99,9 & 30,47 & 0 & 0 & 0 \\
\hline Pipe & 59,9 & 99,9 & 30,47 & 0 & 4921 & 0 \\
\hline Pipe & 61,4 & 140 & 42,67 & 131 & 4593 & 1,6 \\
\hline Pipe & 62,8 & 115 & 35,05 & -82 & 4593 & $-1,0$ \\
\hline Pipe & 65,4 & 22 & 68,58 & 360 & 8530 & 2,4 \\
\hline Side Stream & 65,4 & 225 & 68,58 & 0 & 0 & 0 \\
\hline Pipe & 66,3 & 195 & 59,43 & -98 & 2952 & $-1,91$ \\
\hline Pipe & 75,7 & 200 & 60,96 & 16 & 30839 & 0,03 \\
\hline Pipe & 76,1 & 235 & 71,62 & 114 & 1312 & 5,02 \\
\hline Pipe & 78,2 & 225 & 68,58 & -32 & 6889 & $-0,273$ \\
\hline Pipe & 78,9 & 180 & 54,86 & -147 & 2624 & $-3,225$ \\
\hline
\end{tabular}

Tabla 8. Entrada de secciones, accesorios, elevación y longitud de tubería del tramo Hato Nuevo - La Jagua del Pilar de TGI (Ballena - Barrancabermeja).

\begin{tabular}{ccccccc}
\hline & & & & & \\
\hline Side Stream & 78,9 & 179,9 & 54,8 & 0 & 0 & 0 \\
\hline Compressor & 78,9 & 179,9 & 54,8 & 0 & 0 & 0 \\
\hline Cooler & 78,9 & 179,9 & 54,8 & 0 & 0 & 0 \\
\hline Side Stream & 78,9 & 179,9 & 54,8 & 0 & 0 & 0 \\
\hline Pipe & 80,6 & 179,9 & 54,8 & 0 & 5249 & 0 \\
\hline Pipe & 81,2 & 175,0 & 53,3 & $-16,4$ & 1968 & $-0,47$ \\
Pipe & 83,6 & 225 & 68,5 & 164 & 7874 & 1,1 \\
\hline Pipe & 86,6 & 227 & 69,5 & 9,8 & 9842 & 0,05 \\
\hline Pipe & 89,8 & 250 & 76,2 & 72,1 & 10498 & 0,39 \\
\hline Pipe & 94,4 & 258 & 78,6 & 26,2 & 15255 & 0,09 \\
\hline Pipe & 96,4 & 188 & 57,3 & -229 & 6561 & $-2,0$ \\
\hline Pipe & 111,6 & 178 & 54,2 & -32 & 49704 & $-0,03$ \\
\hline Pipe & 127,3 & 200 & 60,9 & 72 & 51509 & 0,08 \\
\hline Pipe & 138,9 & 200 & 60,9 & 0 & 38385 & 0 \\
\hline Fitting & 138,9 & 200 & 60,9 & 0 & 0 & 0 \\
\hline Pipe & 140,6 & 200 & 60,9 & 0 & 5249 & 0 \\
\hline Side Stream & 140,6 & 200 & 60,9 & 0 & 0 & 0 \\
\hline Pipe & 143,6 & 200 & 60,9 & 0 & 9842 & 0 \\
\hline Pipe & 147,8 & 178 & 54,2 & -72 & 13779 & $-0,3$ \\
\hline Side Stream & 147,8 & 178 & 54,2 & 0 & 0 & 0 \\
\hline Pipe & 159,9 & 175 & 53,3 & -9 & 40026 & $-0,01$ \\
\hline & & & & & & \\
\hline
\end{tabular}

Tabla 9. Entrada de secciones, accesorios, elevación y longitud de tubería del tramo La Jagua del Pilar - Casacará de TGI (Ballena - Barrancabermeja).

\begin{tabular}{ccccccc}
\hline & & & & & \\
\hline Side Stream & 159,9 & 175 & 53,3 & 0 & 0 & 0 \\
Compressor & 159,9 & 175 & 53,3 & 0 & 0 & 0 \\
\hline Cooler & 159,9 & 175 & 53,3 & 0 & 0 & 0 \\
\hline Pipe & 163 & 140 & 42,6 & -114 & 10170 & $-0,64$ \\
\hline Pipe & 169,4 & 130 & 39,6 & $-32,8$ & 20997 & $-0,09$ \\
\hline Side Stream & 169,4 & 130 & 39,6 & 0 & 0 & 0 \\
\hline Pipe & 171,9 & 130 & 39,6 & 0 & 8202 & 0 \\
\hline Pipe & 176,9 & 130 & 39,6 & 0 & 16404 & 0 \\
\hline Side Stream & 176,9 & 130 & 39,6 & 0 & 0 & 0 \\
\hline Pipe & 179,4 & 130 & 39,6 & 0 & 7939 & 0 \\
\hline Pipe & 183,3 & 130 & 39,6 & 0 & 12729 & 0 \\
\hline Pipe & 189,9 & 99,9 & 30,5 & $-98,4$ & 21981 & $-0,25$ \\
\hline Fitting & 189,9 & 99,9 & 30,5 & 0 & 0 & 0 \\
\hline Pipe & 213,9 & 99,9 & 30,5 & 0 & 78740 & 0 \\
\hline Side Stream & 213,9 & 99,9 & 30,5 & 0 & 0 & 0 \\
\hline Pipe & 215,6 & 99,9 & 30,5 & 0 & 5249 & 0 \\
Fitting & 215,6 & 99,9 & 30,5 & 0 & 0 & 0 \\
\hline Pipe & 223,3 & 99,9 & 30,5 & 0 & 25262 & 0 \\
\hline Pipe & 225,6 & 99,9 & 30,5 & 0 & 7545 & 0 \\
\hline Pipe & 228,4 & 109,9 & 33,5 & 32,8 & 9186 & 0,20 \\
\hline Pipe & 235,2 & 99,9 & 30,5 & $-32,8$ & 22309 & $-0,08$ \\
\hline Pipe & 238,9 & 109,9 & 33,5 & 32,8 & 12468 & 0,15 \\
\hline & & & & & & \\
\hline
\end{tabular}


Tabla 10. Entrada de secciones, accesorios, elevación y longitud de tubería del tramo Casacará - Curumaní de TGI (Ballena-Barrancabermeja).

\begin{tabular}{|c|c|c|c|c|c|c|}
\hline 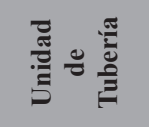 & 竞 & 冚 & 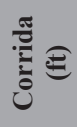 & $\stackrel{\frac{\pi}{2}}{\vdots}$ & 总 & 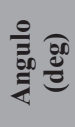 \\
\hline Side Stream & 238,9 & 109,9 & 33,5 & 0 & 0 & 0 \\
\hline Compressor & 238,9 & 109,9 & 33,5 & 0 & 0 & 0 \\
\hline Cooler & 238,9 & 109,9 & 33,5 & 0 & 0 & 0 \\
\hline Side Stream & 238,9 & 109,9 & 33,5 & 0 & 0 & 0 \\
\hline Pipe & 239,9 & 99,9 & 30,5 & $-32,8$ & 3281 & $-0,57$ \\
\hline Pipe & 244,5 & 105 & 32 & 16,4 & 15091 & 0,06 \\
\hline Side Stream & 244,5 & 105 & 32 & 0 & 0 & 0 \\
\hline Pipe & 264,5 & 105 & 32 & 0 & 65288 & 0 \\
\hline Side Stream & 264,5 & 105 & 32 & 0 & 0 & 0 \\
\hline Pipe & 267,9 & 105 & 32 & 0 & 11482 & 0 \\
\hline Fitting & 267,9 & 105 & 32 & 0 & 0 & 0 \\
\hline Pipe & 272,9 & 105 & 32 & 0 & 16076 & 0 \\
\hline Pipe & 277,7 & 99,9 & 30,5 & $-16,4$ & 15912 & $-0,05$ \\
\hline Pipe & 279 & 49,9 & 15,2 & -164 & 4429 & $-2,1$ \\
\hline Side Stream & 279 & 49,9 & 15,2 & 0 & 0 & 0 \\
\hline Pipe & 283 & 49,9 & 15,2 & 0 & 13287 & 0 \\
\hline Pipe & 286 & 49,9 & 15,2 & 0 & 9678 & 0 \\
\hline Pipe & 290 & 60,5 & 18,4 & 34,4 & 16076 & 0,12 \\
\hline Side Stream & 290 & 60,5 & 18,4 & 0 & 0 & 0 \\
\hline Pipe & 291,9 & 60,5 & 18,4 & 0 & 3280 & 0 \\
\hline Fitting & 291,9 & 60,5 & 18,4 & 0 & 0 & 0 \\
\hline Pipe & 294,1 & 60,5 & 18,4 & 0 & 7217 & 0 \\
\hline Pipe & 298,4 & 45 & 13,7 & $-50,8$ & 13779 & $-0,2$ \\
\hline Side Stream & 298,4 & 45 & 13,7 & 0 & 0 & 0 \\
\hline Pipe & 299,9 & 45 & 13,7 & 0 & 5249 & 0 \\
\hline Pipe & 311,8 & 59,9 & 18,2 & 49,2 & 38877 & 0,07 \\
\hline Pipe & 314,9 & 45 & 13,7 & $-49,2$ & 10334 & $-0,27$ \\
\hline Pipe & 316,8 & 55,5 & 16,9 & 34,4 & 5905 & 0,33 \\
\hline Side Stream & 316,8 & 55,5 & 16,9 & 0 & 0 & 0 \\
\hline Pipe & 319,9 & 55,5 & 16,9 & 0 & 10498 & 0 \\
\hline Pipe & 321,6 & 40,5 & 12,3 & $-49,2$ & 5249 & $-0,53$ \\
\hline
\end{tabular}

Tabla 11. Entrada de secciones, accesorios, elevación y longitud de tubería del tramo Curumaní -Norean de TGI (Ballena-Barrancabermeja).

\begin{tabular}{ccccccc}
\hline & & & & \\
\hline Compressor & 321,6 & 40,5 & 12,3 & 0 & 0 & 0 \\
\hline Cooler & 321,6 & 40,5 & 12,3 & 0 & 0 & 0 \\
\hline Pipe & 323,4 & 40,5 & 12,3 & 0 & 6069 & 0 \\
\hline Pipe & 326,7 & 55 & 16,7 & 47,5 & 10826 & 0,2 \\
\hline Pipe & 348,9 & 50,5 & 15,4 & $-14,7$ & 72998 & $-0,01$ \\
\hline Side Stream & 348,9 & 50,5 & 15,4 & 0 & 0 & 0 \\
\hline Pipe & 349,9 & 50,5 & 15,4 & 0 & 3280 & 0 \\
\hline Pipe & 354,1 & 50,5 & 15,4 & 0 & 13615 & 0 \\
\hline
\end{tabular}

\begin{tabular}{ccccccc}
\hline & & & & & \\
\hline Pipe & 354,5 & 40,5 & 12,3 & $-32,8$ & 1148 & $-1,6$ \\
\hline Side Stream & 354,5 & 40,5 & 12,3 & 0 & 0 & 0 \\
\hline Fitting & 354,5 & 40,4 & 12,3 & 0 & 0 & 0 \\
\hline Pipe & 355,2 & 40,4 & 12,34 & 0 & 2296 & 0 \\
\hline Pipe & 355,9 & 115 & 35,05 & 244 & 2624 & 5,3 \\
\hline Pipe & 356,6 & 49,9 & 15,23 & -213 & 1968 & $-6,2$ \\
\hline Pipe & 357,3 & 80,5 & 24,5 & 100 & 2460 & 2,3 \\
\hline Pipe & 359,8 & 49,9 & 15,23 & -100 & 8038 & $-0,7$ \\
\hline Pipe & 363,2 & 60,4 & 18,44 & 34 & 11318 & 0,17 \\
\hline Side Stream & 363,2 & 60,4 & 18,44 & 0 & 0 & 0 \\
\hline Pipe & 394,9 & 49,9 & 15,23 & -34 & 104166 & $-0,01$ \\
\hline Side Stream & 394,9 & 49,9 & 15,23 & 0 & 0 & 0 \\
\hline Pipe & 399,9 & 74,9 & 22,85 & 82 & 16404 & 0,2 \\
\hline Pipe & 411,8 & 109,9 & 33,52 & 114 & 38740 & 0,1 \\
\hline & & & & & & \\
\hline
\end{tabular}

Tabla 12. Entrada de secciones, accesorios, elevación y longitud de tubería del tramo Norean - San Alberto de TGI (Ballena-Barrancabermeja).

\begin{tabular}{ccccccc}
\hline & & & & & & \\
\hline Compressor & 411,8 & 109,9 & 33,52 & 0 & 0 & 0 \\
\hline Cooler & 411,8 & 109,9 & 33,52 & 0 & 0 & 0 \\
\hline Side Stream & 411,8 & 109,9 & 33,52 & 0 & 0 & 0 \\
\hline Pipe & 417,5 & 121,9 & 37,16 & 39,1 & 18700 & 0,12 \\
\hline Pipe & 420 & 146,9 & 44,78 & 82 & 8202 & 0,57 \\
\hline Side Stream & 420 & 146,9 & 44,78 & 0 & 0 & 0 \\
\hline Pipe & 429,9 & 143,8 & 43,85 & -10 & 32808 & $-0,02$ \\
\hline Pipe & 435,9 & 140,2 & 42,73 & -12 & 19685 & $-0,03$ \\
\hline Fitting & 435,9 & 140,2 & 42,73 & 0 & 0 & 0 \\
\hline Pipe & 438,4 & 134,1 & 40,87 & -20 & 8202 & $-0,14$ \\
\hline Pipe & 442,9 & 132,5 & 40,41 & -5 & 14763 & $-0,02$ \\
\hline Pipe & 445,5 & 122,5 & 37,36 & $-32,8$ & 8202 & $-0,23$ \\
\hline Pipe & 462,9 & 124,7 & 38,01 & 7 & 57414 & 0,007 \\
\hline Fitting & 462,9 & 124,7 & 38,01 & 0 & 0 & 0 \\
\hline Pipe & 480 & 121 & 36,9 & -12 & 55774 & $-0,01$ \\
\hline Fitting & 480 & 121 & 36,9 & 0 & 0 & 0 \\
\hline Pipe & 487 & 119,9 & 36,5 & $-3,5$ & 22965 & $-0,01$ \\
\hline Pide Stream & 487 & 119,9 & 36,5 & 0 & 0 & 0 \\
\hline & 498 & 115 & 35,05 & $-16,4$ & 36089 & $-0,02$ \\
\hline
\end{tabular}


Tabla 13. Input de secciones, accesorios, elevación y longitud de tubería del tramo San Alberto - Barrancabermeja de TGI (Ballena - Barrancabermeja).

\begin{tabular}{ccccccc}
\hline & & & & & \\
\hline Compressor & 498 & 115 & 35 & 0 & 0 & 0 \\
\hline Cooler & 498 & 115 & 35 & 0 & 0 & 0 \\
\hline Pipe & 520 & 115 & 35 & 0 & 72178 & 0 \\
\hline Pipe & 521,5 & 159,9 & 48,7 & 147,6 & 4923 & 1,71 \\
\hline Fitting & 521,5 & 159,9 & 48,7 & 0 & 0 & 0 \\
\hline Pipe & 525,5 & 159,9 & 48,7 & 0 & 13123 & 0 \\
\hline Pipe & 526,9 & 124,9 & 38 & -114 & 4922 & $-1,33$ \\
\hline Pipe & 533,5 & 124,9 & 38 & 0 & 21325 & 0 \\
\hline Pipe & 534,9 & 150 & 45,7 & 82 & 4921 & 0,95 \\
\hline Pipe & 544,9 & 150 & 45,7 & 0 & 32808 & 0 \\
\hline Fitting & 544,9 & 150 & 45,7 & 0 & 0 & 0 \\
\hline Pipe & 574 & 150 & 45,7 & 0 & 95144 & 0 \\
\hline Side Stream & 574 & 150 & 45,7 & 0 & 0 & 0 \\
\hline Pipe & 578,7 & 150 & 45,7 & 0 & 15748 & 0 \\
\hline
\end{tabular}

El gasoducto Ballena-Barrancabermeja atraviesa los departamentos de La Guajira, Magdalena, Cesar y Santander, como se mencionó anteriormente. Entre éstos se identifican ciertos municipios claves por su cercanía al recorrido del gasoducto, y otros que a pesar de estar en zonas un poco lejanas al gasoducto tiene cierta influencia sobre este desde el punto de vista climático (Ver Figura 1).

De acuerdo con esto, se establecieron las temperaturas mínimas ambientes en cada porción modelada del gasoducto, las cuales pueden observarse en las Tablas $14,15,16,17,18,19$ y 20 . En cuanto al terreno y los sectores donde la tubería se encuentra enterrada, se utilizaron para el modelado del gasoducto la información suministrada por Peña (Peña, Santos y Pérez, 2004) en sus estudios.

Tabla 14. Entrada de temperatura mínima del tramo Ballena - Hato Nuevo de TGI (Ballena - Barrancabermeja).

\begin{tabular}{ccc}
\hline Unidad de Tubería & $\begin{array}{c}\text { Distancia } \\
\text { (ft) }\end{array}$ & $\begin{array}{c}\text { T Ambiente } \\
\text { (F) }\end{array}$ \\
\hline Pipe & 17,79 & 62,76 \\
Pipe & 26,3 & 63,79 \\
Fitting & 26,3 & 63,79 \\
\hline Pipe & 53,42 & 67,06 \\
\hline Pipe & 53,89 & 67,12 \\
Pipe & 58,49 & 67,67 \\
Fitting & 58,49 & 67,67 \\
\hline
\end{tabular}

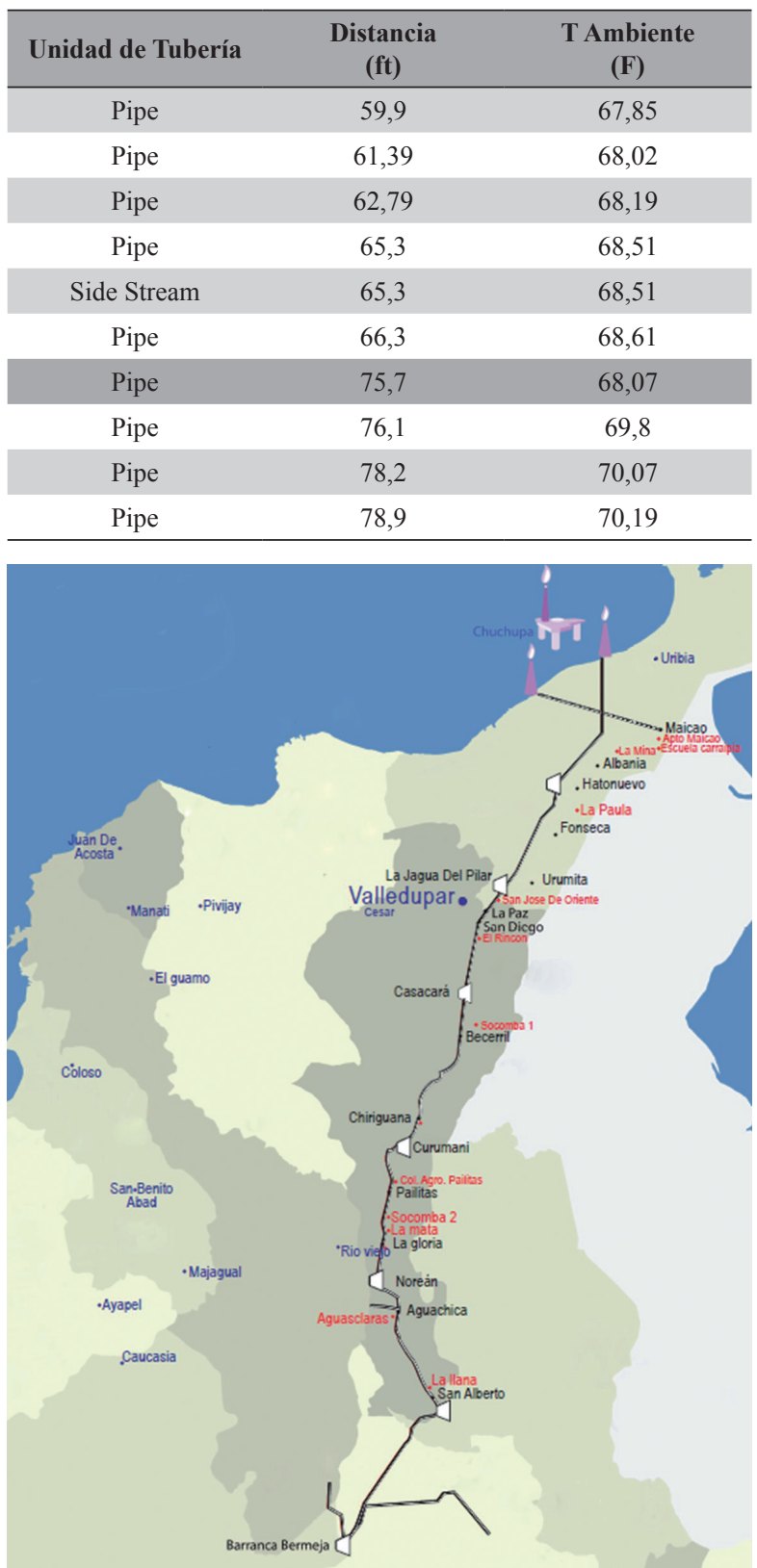

Figura 1. Mapa de municipios que inciden sobre la temperatura ambiente del trayecto del gasoducto TGI.

Fuente: Autores

Tabla 15. Entrada de temperatura mínima del tramo Hato Nuevo - La Jagua del Pilar de TGI (Ballena Barrancabermeja).

\begin{tabular}{ccc}
\hline Unidad de Tubería & $\begin{array}{c}\text { Distancia } \\
(\mathbf{f t})\end{array}$ & $\begin{array}{c}\text { T Ambiente } \\
(\mathbf{F})\end{array}$ \\
\hline Side Stream & 78,9 & 63,21 \\
\hline Compressor & 78,9 & 63,21 \\
Cooler & 78,9 & 63,21 \\
Side Stream & 78,9 & 63,21 \\
Pipe & 80,6 & 63,21 \\
\hline
\end{tabular}




\begin{tabular}{ccc}
\hline Unidad de Tubería & $\begin{array}{c}\text { Distancia } \\
(\mathbf{f t})\end{array}$ & $\begin{array}{c}\text { T Ambiente } \\
(\mathbf{F})\end{array}$ \\
\hline Pipe & 81,2 & 63,24 \\
\hline Pipe & 83,6 & 63,37 \\
Pipe & 86,6 & 63,53 \\
\hline Pipe & 89,8 & 63,7 \\
\hline Pipe & 94,4 & 63,95 \\
\hline Pipe & 96,4 & 64,07 \\
\hline Pipe & 111,6 & 65,72 \\
\hline Pipe & 127,3 & 66,35 \\
\hline Pipe & 138,9 & 66,44 \\
\hline Fitting & 138,9 & 66,44 \\
Pipe & 140,6 & 67,49 \\
\hline Side Stream & 140,6 & 67,49 \\
\hline Pipe & 143,6 & 67,66 \\
\hline Pipe & 147,8 & 66,56 \\
Side Stream & 147,8 & 66,56 \\
Pipe & 159,9 & 68,7 \\
\hline & & \\
\hline & & 6 \\
\hline
\end{tabular}

Tabla 16. Entrada de temperatura mínima del tramo La Jagua del Pilar - Casacará de TGI (Ballena - Barrancabermeja).

\begin{tabular}{|c|c|c|}
\hline Unidad de Tubería & $\begin{array}{l}\text { Distancia } \\
\text { (ft) }\end{array}$ & $\begin{array}{c}\text { T Ambiente } \\
\text { (F) }\end{array}$ \\
\hline Side Stream & 159,9 & 66,56 \\
\hline Compressor & 159,9 & 66,56 \\
\hline Cooler & 159,9 & 66,56 \\
\hline Pipe & 163 & 66,56 \\
\hline Pipe & 169,5 & 67,63 \\
\hline Side Stream & 169,5 & 67,63 \\
\hline Pipe & 171,9 & 69,21 \\
\hline Pipe & 176,9 & 63,98 \\
\hline Side Stream & 176,9 & 63,98 \\
\hline Pipe & 179,4 & 67,86 \\
\hline Pipe & 183,3 & 69,19 \\
\hline Pipe & 189,9 & 70,65 \\
\hline Fitting & 189,9 & 70,65 \\
\hline Pipe & 213,9 & 71,6 \\
\hline Side Stream & 213,9 & 71,6 \\
\hline Pipe & 215,6 & 71,07 \\
\hline Fitting & 215,6 & 71,07 \\
\hline Pipe & 223,3 & 72,21 \\
\hline Pipe & 225,6 & 72,6 \\
\hline Pipe & 228,4 & 70,8 \\
\hline Pipe & 235,2 & 68,98 \\
\hline Pipe & 238,9 & 67,8 \\
\hline
\end{tabular}

Tabla 17. Entrada de temperatura mínima del tramo Casacará - Curumaní de TGI (Ballena - Barrancabermeja).

\begin{tabular}{ccc}
\hline Unidad de Tubería & $\begin{array}{c}\text { Distancia } \\
(\mathbf{f t})\end{array}$ & $\begin{array}{c}\text { T Ambiente } \\
(\mathbf{F})\end{array}$ \\
\hline Side Stream & 238,9 & 66,2 \\
Compressor & 238,9 & 66,2 \\
Cooler & 238,9 & 66,2 \\
\hline Side Stream & 238,9 & 66,2 \\
\hline
\end{tabular}

\begin{tabular}{|c|c|c|}
\hline Unidad de Tubería & $\begin{array}{c}\text { Distancia } \\
\text { (ft) }\end{array}$ & $\begin{array}{c}\text { T Ambiente } \\
\text { (F) }\end{array}$ \\
\hline Pipe & 239,9 & 66,2 \\
\hline Pipe & 244,5 & 66,85 \\
\hline Side Stream & 244,5 & 66,85 \\
\hline Pipe & 264,4 & 69,15 \\
\hline Side Stream & 264,4 & 69,15 \\
\hline Pipe & 267,9 & 69,98 \\
\hline Fitting & 267,9 & 69,98 \\
\hline Pipe & 272,8 & 70,24 \\
\hline Pipe & 277,7 & 70,19 \\
\hline Pipe & 279 & 68,15 \\
\hline Side Stream & 279 & 68,15 \\
\hline Pipe & 283,1 & 66,2 \\
\hline Pipe & 286 & 65,5 \\
\hline Pipe & 290,9 & 67,9 \\
\hline Side Stream & 290,9 & 67,9 \\
\hline Pipe & 291,9 & 68,1 \\
\hline Fitting & 291,9 & 68,1 \\
\hline Pipe & 294,1 & 64,3 \\
\hline Pipe & 298,3 & 62,3 \\
\hline Side Stream & 298,3 & 62,3 \\
\hline Pipe & 299,9 & 65,8 \\
\hline Pipe & 311,8 & 67,9 \\
\hline Pipe & 314,9 & 68,1 \\
\hline Pipe & 316,7 & 66,2 \\
\hline Side Stream & 316,7 & 66,2 \\
\hline Pipe & 319,9 & 67,63 \\
\hline Pipe & 321,5 & 68,15 \\
\hline
\end{tabular}

Tabla 18. Entrada de temperatura mínima del tramo Curumaní - Norean de TGI (Ballena - Barrancabermeja).

\begin{tabular}{|c|c|c|}
\hline Unidad de Tubería & $\begin{array}{l}\text { Distancia } \\
\text { (ft) }\end{array}$ & $\begin{array}{l}\text { T Ambiente } \\
\text { (F) }\end{array}$ \\
\hline Compressor & 321,59 & 66,2 \\
\hline Cooler & 321,59 & 66,2 \\
\hline Pipe & 323,44 & 66,2 \\
\hline Pipe & 326,74 & 67,63 \\
\hline Pipe & 348,99 & 69,21 \\
\hline Side Stream & 348,9 & 69,21 \\
\hline Pipe & 349,9 & 68,15 \\
\hline Pipe & 354,14 & 68,59 \\
\hline Pipe & 354,49 & 69,6 \\
\hline Side Stream & 354,49 & 69,6 \\
\hline Fitting & 354,49 & 69,6 \\
\hline Pipe & 355,1 & 69,15 \\
\hline Pipe & 355,99 & 69,98 \\
\hline Pipe & 356,59 & 70,24 \\
\hline Pipe & 357,34 & 68,34 \\
\hline Pipe & 359,79 & 68,59 \\
\hline Pipe & 363,24 & 69,6 \\
\hline Side Stream & 363,24 & 69,6 \\
\hline Pipe & 394,99 & 67,63 \\
\hline Side Stream & 394,99 & 67,63 \\
\hline Pipe & 399,99 & 64,4 \\
\hline Pipe & 411,8 & 66,68 \\
\hline
\end{tabular}


Tabla 19. Entrada de temperatura mínima del tramo Norean - San Alberto de TGI (Ballena - Barrancabermeja).

\begin{tabular}{ccc}
\hline Unidad de Tubería & $\begin{array}{c}\text { Distancia } \\
\text { (ft) }\end{array}$ & $\begin{array}{c}\text { T ambiente } \\
\text { (F) }\end{array}$ \\
\hline Compressor & 411,8 & 70,1 \\
\hline Cooler & 411,8 & 70,1 \\
\hline Side Stream & 411,8 & 70,1 \\
\hline Pipe & 417,5 & 70,1 \\
\hline Pipe & 420 & 69,6 \\
\hline Side Stream & 420 & 69,6 \\
\hline Pipe & 429,9 & 69,21 \\
\hline Pipe & 435,9 & 67,63 \\
\hline Fitting & 435,9 & 67,63 \\
\hline Pipe & 438,4 & 70,65 \\
\hline Pipe & 442,9 & 71,11 \\
\hline Pipe & 445,4 & 70,1 \\
\hline Pipe & 462,9 & 68,59 \\
\hline Fitting & 462,9 & 68,59 \\
\hline Pipe & 480 & 67,43 \\
\hline Fitting & 480 & 67,43 \\
\hline Pipe & 487 & 68,56 \\
\hline Side Stream & 487 & 68,56 \\
\hline Pipe & 498 & 68,24 \\
\hline & & \\
\hline & & \\
\hline
\end{tabular}

Tabla 20. Entrada de temperatura mínima del tramo San Alberto - Barrancabermeja de TGI (Ballena Barrancabermeja).

\begin{tabular}{ccc}
\hline Unidad de Tubería & $\begin{array}{c}\text { Distancia } \\
(\mathbf{f t})\end{array}$ & $\begin{array}{c}\text { T Ambiente } \\
(\mathbf{F})\end{array}$ \\
\hline Compressor & 498 & 68,59 \\
\hline Cooler & 498 & 68,59 \\
\hline Pipe & 520 & 68,59 \\
\hline Pipe & 521,5 & 68,15 \\
\hline Fitting & 521,5 & 68,15 \\
\hline Pipe & 525,5 & 69,66 \\
\hline Pipe & 526,9 & 67,67 \\
\hline Pipe & 533,5 & 66,79 \\
\hline Pipe & 534,9 & 66,98 \\
\hline Pipe & 544,9 & 67,43 \\
\hline Fitting & 544,9 & 67,43 \\
\hline Pipe & 574 & 68,2 \\
\hline Side Stream & 574 & 68,2 \\
\hline Pipe & 578 & 68,91 \\
\hline
\end{tabular}

\section{RESULTADOS DE LA SIMULACIÓN}

Para cada gasoducto por separado, y cada escenario de evaluación de los mismos, se generaron los perfiles topográficos, de presión, temperatura del gas en el gasoducto, temperatura mínima ambiente, y ruta operacional del gas (Presión vs. Temperatura). De estos se concluyó que para todos los escenarios propuestos, en ambos gasoductos, no se generaron líquidos a las condiciones establecidas. Además se establecieron los nodos críticos para la evaluación del efecto JouleThomson en los City Gates.

\subsection{TGI (BALLENA- \\ BARRANCABERMEJA) \\ - ESCENARIO I (260 \\ MMSCFD Y 1200 PSIG)}

En este primer escenario se consideraron las caídas de presión experimentadas en el gasoducto en su recorrido y las presiones mínimas y máximas de succión permisibles en las estaciones de compresión (Mapa red nacional de gasoductos: gasoducto ballena - Barrancabermeja). De esto se dedujo que las 6 estaciones existentes deben estar encendidas y funcionando, dado que el caudal es suficientemente alto para generar turbulencia suficiente y por ende altas caídas de presión en la línea.

Perfil Topográfico. Teniendo en cuenta la información de longitud de tramos y elevación punto a punto del modelo de simulación, se construyó el siguiente perfil topográfico, el cual aplica indiscriminadamente para ambos escenarios de evaluación del gasoducto de TGI (Ver Figura 2).

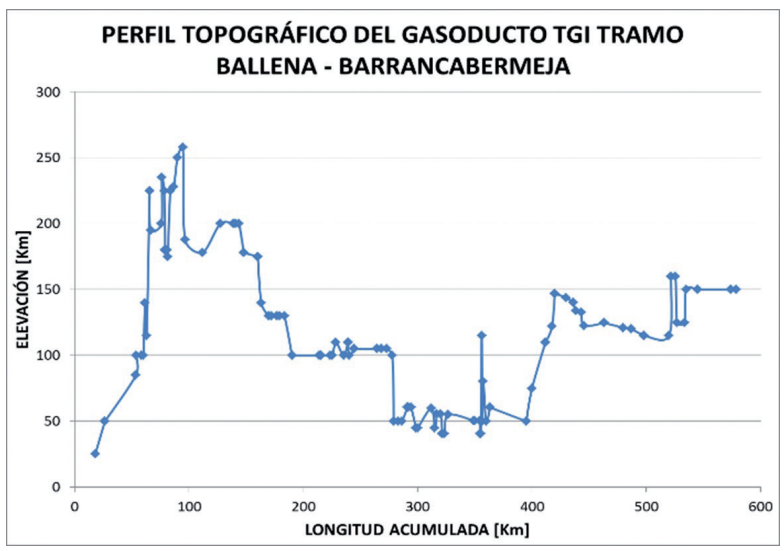

Figura 2. Perfil Topográfico del gasoducto TGI. Fuente: Autores

Perfil de Presión. Se construyó el perfil de presión con los resultados de la simulación (Ver Figura 3). En este perfil se identifican las caídas de presión abruptas debido al flujo muy turbulento a lo largo de los tramos, y el ascenso que ocurre en las estaciones de compresión.

Perfil de Temperaturas. Se elaboró el perfil de temperaturas (Ver Figura 4) incluyendo la temperatura mínima ambiente, la temperatura del gas fluyendo en 
el gasoducto, los valores tentativos de $\operatorname{HCDP}(62,60 \mathrm{y}$ $\left.55^{\circ} \mathrm{F}\right)$ y el valor actual de $\operatorname{HCDP}\left(45^{\circ} \mathrm{F}\right)$ con los datos resultantes de la simulación.

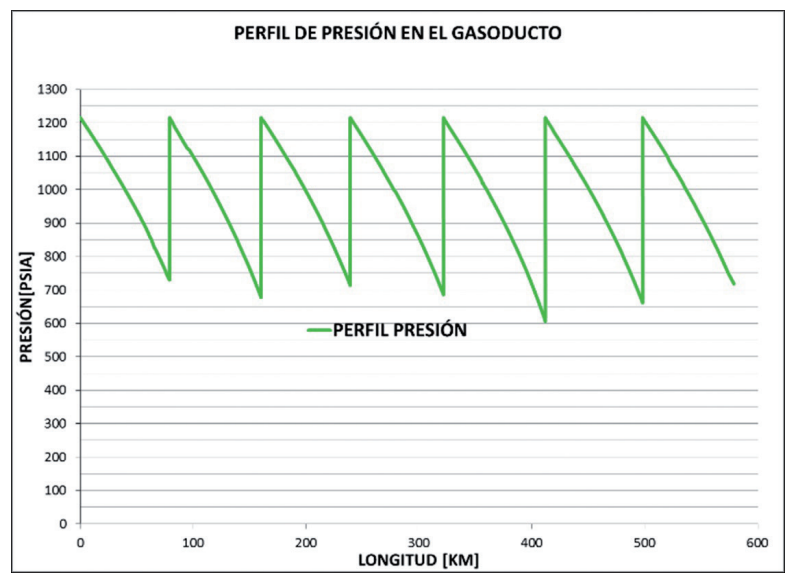

Figura 3. Perfil de presión del gasoducto TGI para el escenario I (260MMSCFD).

Fuente: Autores.

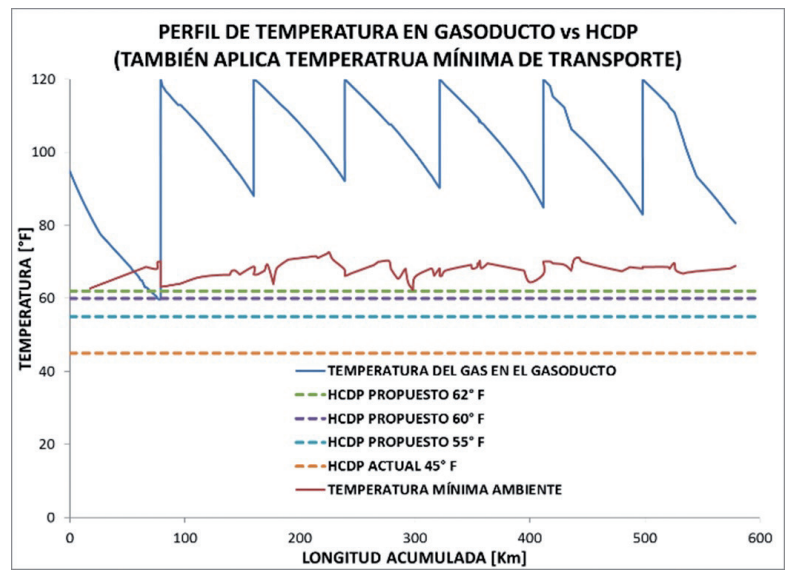

Figura 4. Perfil de temperaturas de TGI para el escenario I (260MMSCFD).

Fuente: Autores.

Dado que por convención en Colombia se ha fijado la temperatura mínima de entrega igual al valor máximo de HCDP, estos perfiles de temperatura en comparación con las líneas de calidad de HCDP establecidas, dan un indicativo del cumplimiento de la norma por temperaturas mínimas. En este caso particular (escenario crítico que involucra máximo caudal y a su vez que en un instante de tiempo determinado todo el trayecto que recorre el gasoducto experimente a la vez las temperaturas mínimas de los municipios aledaños) se puede concluir que para estas condiciones, sin lo equipos de calentamiento adecuados en el sector cercano a la estación de compresión de Hato Nuevo, se condensarían líquidos HC para gases con HCDP mayores a $60^{\circ} \mathrm{F}$.
Ruta operacional y envolvente de fase. También se realizó un análisis de la ruta operacional del gasoducto conformada por los valores de presión y temperatura del gas de los perfiles anteriores en cada punto del trayecto. Estos datos se compararon con las envolventes del Gas de Ballena y los tres gases genéricos que representan los valores tentativos de HCDP (Ver Figura 5).

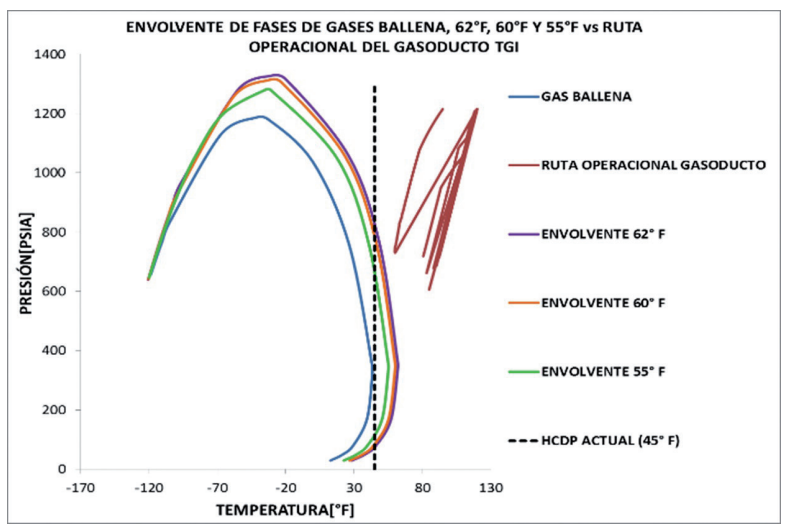

Figura 5. Evaluación de la ruta operacional con las envolventes para TGI escenario I (260MMSCFD). Fuente: Autores.

\subsection{TGI (BALLENA-}

\section{BARRANCABERMEJA) \\ - ESCENARIO II $(76,4$ MMSCFD Y 1068 PSIG)}

En el segundo escenario, bajo consideración de las caídas de presión experimentadas en el gasoducto en su recorrido y las presiones mínimas y máximas de succión permisibles en las estaciones de compresión compresión (Mapa red nacional de gasoductos: gasoducto ballena - Barrancabermeja), se decidió apagar 5 estaciones de compresión con problemas operacionales por presiones de entrada superiores a la máxima presión de succión permisible, además de ser práctica general en la operación a diario para ahorro energético. Se dejó en funcionamiento total la estación de Curumaní, a la cual el gas llegaba con presión dentro del rango operativo de presión de succión.

Perfil de presión. Se construyó el perfil de presión con los resultados de la simulación (Ver Figura 6). En este perfil se identifican caídas de presión más suaves en comparación con el escenario I, evidenciado los aspectos de presión de succión comentados anteriormente.

Perfil de Temperaturas. Se elaboró el perfil de temperaturas (Ver Figura 7) incluyendo la temperatura mínima ambiente, la temperatura del gas fluyendo en el gasoducto, los valores tentativos de $\operatorname{HCDP}(62,60 \mathrm{y}$ 
$\left.55^{\circ} \mathrm{F}\right)$ y el valor actual de $\operatorname{HCDP}\left(45^{\circ} \mathrm{F}\right)$ con los datos resultantes de la simulación.

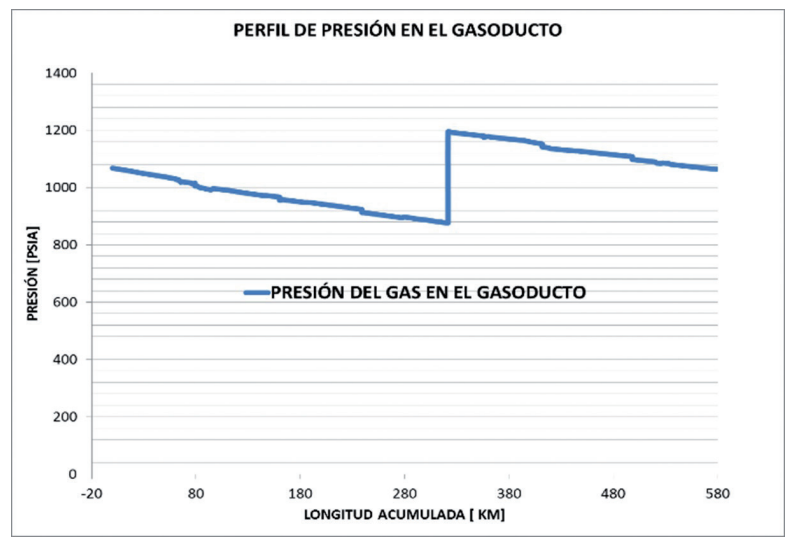

Figura 6. Perfil de presión del gasoducto TGI para el escenario II (76,4MMSCFD).

Fuente: Autores.

Dado que por convención en Colombia se ha fijado la temperatura mínima de entrega igual al valor máximo de HCDP, estos perfiles de temperatura en comparación con las líneas de calidad de HCDP establecidas, dan un indicativo del cumplimiento de la norma por temperaturas mínimas. En este caso, a diferencia del escenario anterior, los gases analizados en el modelo de simulación no condensan líquidos $\mathrm{HC}$ para ningún HCDP propuesto.

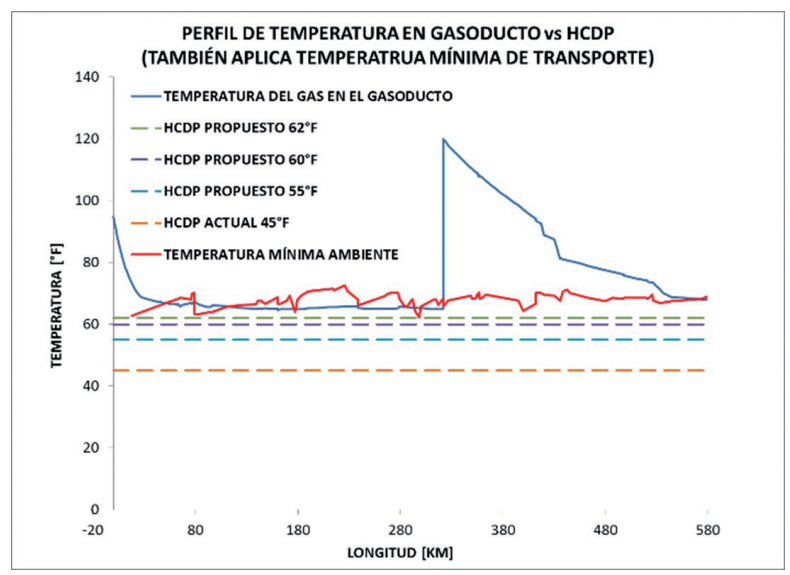

Figura 7. Perfil de temperaturas de TGI para el escenario II (76,4MMSCFD).

Fuente: Autores.

Ruta operacional y envolvente de fases. Se realizó un análisis de la ruta operacional del gasoducto conformada por los valores de presión y temperatura del gas de los perfiles anteriores en cada punto del trayecto. Estos datos se compararon con las envolventes del Gas de Ballena y los tres gases genéricos que representan los valores tentativos de HCDP (Ver Figura 8).

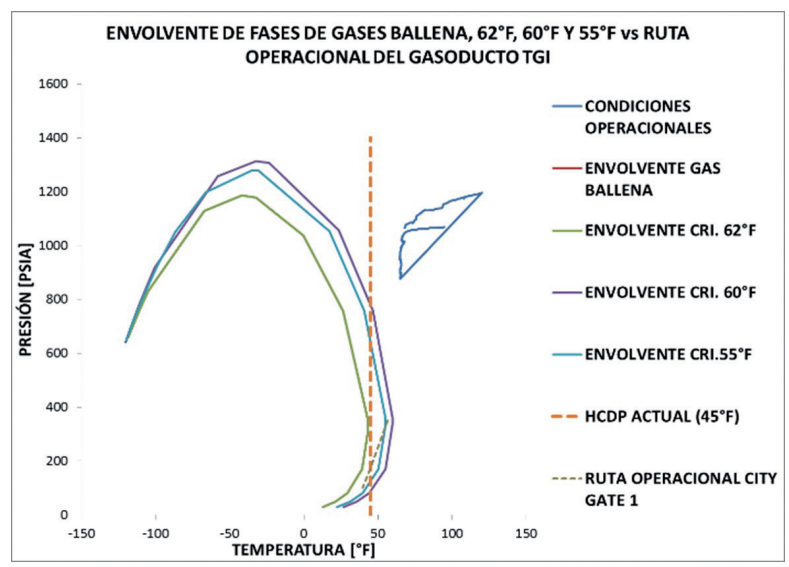

Figura 8. Evaluación de la ruta operacional con las envolventes para TGI escenario II (76,4MMSCFD). Fuente: Autores.

\section{CONCLUSIONES}

El comportamiento termodinámico del gas natural está directamente relacionado con la composición del gas, y dado que cada gas tiene una composición única, deben tener especificaciones diferentes. Adicional a esto está demostrado que el entorno climático de la región afecta el punto de rocío hidrocarburo del gas natural, por eso en una región con estaciones diferentes o diferentes periodos climáticos es conveniente que tenga múltiple punto de rocío, para evitar problemas operacionales.

Si bien un valor único de HCDP genera uniformidad en el mercado, no hace diferencia entre pisos térmicos por lo que en zonas más cálidas podría generar inconvenientes operacionales.

La modalidad contractual, sería inviable para el caso de Colombia, ya que no hay uniformidad de criterios entre productores, transportadores y entes reguladores.

La responsabilidad de la calidad del gas en los City Gate corresponde al operador del gasoducto o transportador.

El valor de $\mathrm{HCDP}$ de $60^{\circ} \mathrm{F}$ permite operaciones óptimas en la línea de transporte sin tendencia a condensar líquidos, teniendo en cuenta que no se generaron líquidos bajo esquemas de evaluación crítica y de operación normal para ambos gasoductos.

Los nodos correspondientes a los puntos antes de las estaciones de compresión de TGI (Excepto Hato Nuevo, San Alberto y Salida a Barrancabermeja), no generan líquidos a presión alguna de regulación por encima de los 100 psig, para todos los HCDP. 
Para los valores de HCDP propuestos de 62 y $60^{\circ} \mathrm{F}$, se requieren instalar calentadores en los puntos cercanos a la entrada a estaciones de compresión de San Alberto y Barrancabermeja para el Escenario I de TGI, y en City Gates cercanos a la entrada al gasoducto para el Escenario II de TGI.

Debe instalarse calentadores con capacidad suficiente de compensar el descenso de temperatura en todos los City Gates después de zonas cercanas a la entrada al gasoducto, para cumplir con el reglamento que regula el transporte de gas en cuanto a temperatura mínima de entrega, a condiciones de presiones de salida de la estación de regulación desde 340 psig.

\section{REFERENCIAS}

1. Acer - Agency for the Cooperation of Energy Regulators. (2011). Framework Guidelines on Interoperability Rules for European Gas Transmission Networks.

2. Alemania. (2013). GRTgaz. Gas Specification.

3. Argentina. Ente Nacional Regulador del Gas ENARGAS. (2005). Ley24.076. Reglamentación de las Especificaciones de Calidad de Gas Natural. Buenos Aires.

4. Belgica. (2004). Energy Charter Secretariat. $L N G$ and Natural Gas Quality Standards.

5. Bolivia. Superintendencia de Hidrocarburos. (2007). Resolución Administrativa No. 0433/2007. Términos y Condiciones Generales de Servicio de Gas Transboliviano. La Paz.

6. BP. International Gas Union. (2011). Guidebook to Gas Interchangeability and Gas Quality.

7. Brasil. Agencia Nacional del Petróleo - ANP. (2002). Ordenanza No. 104 de 2002. Establece las Especificaciones del gas natural, de origen nacional o importado, que se comercialicen en el país. Estatus: Derogada.

8. Brasil. (2008). Agencia Nacional del Petróleo - ANP. Resolución No. 16 de 2008. Establece las Especificaciones del gas natural, de origen nacional o importado, que se comercialicen en el país. Estatus: Actual.

9. Canadá. (2013). TransCanada. Gas Quality Specifications.
10. Canadá. (2006). TransGas. Standard Natural Gas Specifications.

11. Ecuador. (2009). Instituto Ecuatoriano de Normalización. Norma Técnica Ecuatoriana NTE INEN 2 489:2009. Gas Natural. Requisitos. Quito.

12. España. (2011). Enagas. Gestión Tecnica del Sistema. Calidad del Gas.

13. Great Southern Press. Australia. Recuperado de: https://www.gs-press.com.au/\#. Consultado el: 05 de noviembre de 2013.

14. Great Australian Pipeline Industry Association. Australia. Recuperado de: http://www.apga. org.au/. Consultado el: 05 de noviembre de 2013.

15. Irlanda. (2008). Bord Gais Nestworks. Report on Gas Quality Arrangements in Republic of Ireland.

16. Lituania. (2012). Lithuanian Energy Institute. Natural Gas Quality Changes Analysis and Estimation of Transmission System Values.

17. Marcogaz. (2003). 1ST Position Paper on European Gas Quality Specifications. Gas Quality. UTIL03-06. 18. Marcogaz. National Situations Regarding Gas Quality. UTIL-GQ-02-19. 2002.

18. Mexico. (2010). Comisión Reguladora de Energía. Secretaría de Energía. Norma Oficial Mexicana NOM-001-SECRE-2010. Especificaciones del Gas Natural. Mexico D. F.

19. Mapa Red Nacional de Gasoductos: Gasoducto Ballena - Barrancabermeja. Transportadora de Gas Natural Internacional - TGI S.A. E.S.P. www.tgi.com.co.

20. Peña, H., Santos, N. y Pérez, J. (2004). Estudio experimental, caracterización composicional detallada, ajuste de la ecuación de estado y generación de las envolventes de fase de las corrientes de carga a los gasoductos Centragas y Promigas. Universidad Industrial de Santander (UIS). Bucaramanga, Santander (Colombia).

21. Nueva Zelanda. Gas Industry Company Limited. Recommendation to the Minister of Energy by 
the Gas Industry Co. on the Specification for Reticulated Natural Gas.

22. Nueva Zelanda. (1999). National Regulation. NZS 5442:1999.

23. Poyry. (2011). GL Noble Denton. Study on Interoperability - Gas Quality Harmonisation - Cost Benefir Analysis.

24. Rusia. (2008). Gazprom Export. Storage
Specification. Moscow.

25. United Kingdom. (2013). Interconnector. Guide to Gas Quality Requirements.

26. United Kingdom. (2013). Premier Transmission Limited. Transportation Code.

27. USA. (2004). The University of Center For Energy Economics. Interstate Natural Gas - Quality Specifications and Interchangeability. Austin.

Recepción: 27 de septiembre de 2016

Aceptación: 3 de noviembre de 2016 